\title{
Effect of Coenzyme Q10 Supplementation on Exercise-Induced Response of Oxidative Stress and Muscle Damage Indicators in Male Runners
}

\author{
Mostafa Armanfar ${ }^{1, *}$; Afshar Jafari ${ }^{1}$; Gholam Reza Dehghan ${ }^{2}$ \\ ${ }^{1}$ Department of Sport Physiology, Faculty of Physical Education and Sport Sciences, University of Tabriz, Tabriz, IR Iran \\ ${ }^{2}$ Department of Biochemistry, Faculty of Natural Sciences, University of Tabriz, Tabriz, IR Iran \\ ${ }^{*}$ Corresponding author: Mostafa Armanfar, Department of Sport Physiology, Faculty of Physical Education and Sport Sciences, University of Tabriz, Tabriz, IR Iran. \\ E-mail: mostafaarmanfar@yahoo.com
}

Received: April 28, 2014; Accepted: June 10, 2014

\begin{abstract}
Background: Heavy exercise cause muscle damage associated with very-high production of free radicals. The aim of this research was to evaluate the effect of acute and 14-day coenzyme Q10 supplementation on oxidative stress and muscle damage indicator in elite male middle-distance runners.

Materials and Methods: In this experimental study, 18 male runners in a randomly and double-blind design were allocated in two equal groups: supplement Q10 group ( $\mathrm{n}=9$, coenzyme Q10: $5 \mathrm{mg} / \mathrm{kg} /$ day) and placebo group ( $\mathrm{n}=9$, dextrose: $5 \mathrm{mg} / \mathrm{kg} / \mathrm{day}$ ). Before and after supplementation acute and 14 days period (first and 14th day supplementation), all subjects were participated in a training like running (competitive 3000 meters). Blood samples were obtained in the 4 phases: one hour before and 18 - 24 hours after two running protocols. Malondialdehyde (MDA), total antioxidation capacity(TAC) and lactate dehydrogenase (LDH) were analyzed.

Results: Acute (1 day) and 14 days (short-term) coenzyme Q10 administration have not significant effect on basal parameters. The 14 days coenzyme Q10 supplementation induced increase of the basal plasma total anti-oxidative capacity $(\mathrm{P}<0.05)$. The short-term coenzyme Q10 supplementation attenuated the exercise-induced increase in response of MDA in male group Q10 $(\mathrm{P}<0.05)$. However, the acute and short-term coenzyme Q10 supplementation had not any significant effect on the exercise-induced increase response of total serum LDH. Conclusions: This research suggests that the 14-day coenzyme Q10 supplementation is more effective than the acute supplementation to overcome the exercise-induced adverse responses in some oxidative, and biochemical parameters. Therefore, short-term coenzyme Q10 supplementation is recommended to reduce exercise-induced adverse consequences.
\end{abstract}

Keywords: Runners; Coenzyme Q10; Oxidative stress; Muscle damage

\section{Background}

In the past decade, high-intensity competitive sports such as the marathon; long-distance running, middledistance running and triathlon are becoming progressively popular around the world [1-3]. The vigorous exercise-induced muscle damage has been associated with a high degree of oxidative stress $[4,5]$. Thus, this damage may be reduced by optimizing nutrition, especially by increasing the dietary content of oral antioxidants $[5,6]$. Oxidative stress and its consequences results the damage of biological components, e.g. proteins, lipids and genetic elements, and is related to the prevalence of some diseases $[3,7]$. Therefore, it is important to increase antioxidant capacity in tissues to scavenge reactive oxygen species (ROS) produced by strenuous exercise. Recent studies suggests that supplementation of certain antioxidants are practicable for physically active individuals to prevent exerciseinduced muscle damage and recover from tiredness faster $[3,7]$. Supplementation of exogenous antioxidants, such as vitamin C, vitamin E, and carotenoids, has prevented intense exercise-induced oxidative damage in humans and rats $[1,3,8,9]$. Influence of coenzyme Q10 (CoQ10) supple- mentation on exercise-induced muscle damage and oxidative stress has been examined in rats and in humans, however the existing data are limited and inconsistent [3, $10,11]$. Besides, the effect of CoQ10 supplementation on vigorous exercise-related oxidative stress has been unknown. Coenzyme Q10, a component of the electron transport chain in mitochondria is essential for ATP synthesis, especially in tissues with high metabolic demand such as muscle tissue during intensive exercise. CoQ10 acts as a redox electron transporter in the mitochondria $[5,12]$. For many years, this mitochondrial component has been used as a dietary supplement intended to improve optimal health by trapping free radicals and the interest for this molecule comes from the fact of this role as a redox link in the mitochondrial electron transport chain, where also has important antioxidant properties under lipophilic conditions [5]. The data available have provided a direct link between physical performance and blood and muscle tissue CoQ10 levels $[5,13]$. However, most of these studies are focused mainly in the exercise performance and radical-scavenging activity of CoQ10 during low-intensity

Copyright (C) 2015, Zahedan University of Medical Sciences. This is an open-access article distributed under the terms of the Creative Commons Attribution-NonCommercial 4.0 International License (http://creativecommons.org/licenses/by-nc/4.0/) which permits copy and redistribute the material just in noncommercial usages, provided the original work is properly cited. 
exercise [5, 13], being scarce the studies about the influence of CoQ10 supplementation during the performance of high-intensity strenuous exercise, oxidative stress and muscle damage [5]. Therefore, the purpose of this study was to determine the effect of supplementation acute (one day) and short-term (14 days) CoQ10 supplementation ( $5 \mathrm{mg} / \mathrm{kg} /$ day) on the heavy exercise-induced muscle injury and oxidative stress in the middle-distance (following a competition run 3000 meters).

\section{Materials and Methods}

In this experimental study, 18 male middle-distance runners (aged $19.9 \pm 2.64$ years; height $177.6 \pm 2.3 \mathrm{~cm} ; \mathrm{VO}_{2} \max$ $60.6 \pm 3.9)$ in a randomized and double-blind design were divided in two equal Q10 supplement and placebo group. None of the subjects had ingested Q10 or any other dietary supplements (such as caffeine, ginger, ibuprofen, etc.) before initiation of the study. In addition, they have no past history of heart, kidney disease and diabetes or any physical damage and problems. Study was approved by the research ethics committee of Tabriz University of Medical Sciences and designed with two groups (experimental and control) with repeated measures (four blood sampling) a double-blind study. Sample size for each of the two groups with regard to study design and results of previous studies, were estimated seven [14]. However, in order to prevent possible losses during the process of research subjects and the anthropometric indices and aerobic power, sample size for each group was 9 people.

Finally, with regard to maximal oxygen consumption test (Bruce), percentage of body fat and some blood parameters (hemoglobin and hematocrit) were divided into nine groups matched in homogenize supplement CoQ10 and placebo were replaced.

The subjects were randomly assigned to either the Q10 (5 mg/kg/day Q10) or placebo (5 mg/kg/day dextrose). Both treatments were effervescent capsules, pre-packaged to be identical in taste and appearance. Each group ingested one capsule three times per day at regular interval (breakfast, lunch and dinner) for one day and 14 days [13]. The CoQ10 dosage, based on previous studies and least amount of CoQ10 plasma levels required for promotion or deal with the loss of a relatively intense aerobic activity intended [15-17].

Before and after acute and short-term supplementation period, all subjects participated in a 3000 meters running competition. Three thousand meter to competitive running was performed after a general warm-up. In addition, it was assumed that running the 3000 meters running competition with relatively high intensity and hard work as a fuel pressure should cause significant changes in markers of inflammation in the elite male distance runners $[3,18,19]$.

Plasma malondialdehyde (MDA), total antioxidan capacity (TAC) and lactate dehydrogenase (LDH) were obtained in the two phases: before and immediately after two running protocols. Blood samples were obtained in the four phases: one hour before and 18 - 24 hours after two running protocols. Blood samples were obtained from subjects antecubital vein using venipuncture $(5 \mathrm{~mL}$; made by SUHA Co) before and 24 - 18 hours after acute (one day) and short-term (14-day) supplementation protocol. All were measured in hours 11 - $9 \mathrm{pm}$, the temperature of $22-24^{\circ} \mathrm{C}$, humidity 50-60\%, the ventilation and lighting identical. In addition, subjects performed 24 hours before the test, avoid doing any heavy physical activity and meal (breakfast) before the test was similar. In addition, prior to the second blood sampling, subjects' diet was controlled using 24 hours dietary recalls [15]. The distribution normality of variables was tested by the Kolmogorov-Smirnov test. Descriptive statistics were expressed as mean \pm SD. Change any of the parameters (mean and standard deviation) of four stages repeated ANOVA and post hoc tests Bonferuni analysis. Differences between groups were determined by t-test. All statistical analyses were performed using the SPSS-18 software (SPSS Inc., Chicago, IL, USA). The significance level was set at $\mathrm{P}<0.05$.

\section{Results}

The mean and standard deviation of individual characteristics and hemodynamic parameters in both groups before the exercise protocol are given in Table 1. All subject reported adherence to the experimental protocol and complete ingestion of the supplement. There were no differences among groups at the beginning of the study for physical characteristics (Table 1) and biochemical and hemodynamic variables (Table 2). Acute supplementation (1 day) has no significant effect on the variables baseline levels. Short-term supplementation (14-days) cause significantly increased total antioxidant (TAC) baseline levels $(\mathrm{P}<0.05)$. Acute supplementation has no significant effect on variables significantly increased (MDA and LDH) or decreased (TAC) after exercise protocol. The reducing exercise-induced changes range of the plasma TAC following the short-term CoQ10 supplementation attenuated the exercise induced increase in response of plasma $\operatorname{MDA}(\mathrm{P}<0.05)$. However, the acute and short-term CoQ10 supplementation had not any significant effect on the exercise-induced increase response of total serum LDH.

\begin{tabular}{|c|c|c|}
\hline Variables & $\mathrm{CoQ10}^{\mathrm{C}}$ & Placebo $^{C}$ \\
\hline Age, y & $19.4 \pm 2.4$ & $19.9 \pm 2.6$ \\
\hline Height, cm & $175.6 \pm 5.6$ & $177.8 \pm 6.1$ \\
\hline Weight, kg & $68.4 \pm 6.4$ & $68.6 \pm 6.5$ \\
\hline BMI, kg.m² & $22.1 \pm 1.5$ & $21.6 \pm 32.2$ \\
\hline Body fat, \% & $8.9 \pm 1.9$ & $8.9 \pm 1.3$ \\
\hline VO2 max, $\mathrm{mL} / \mathrm{kg}^{-1} / \mathrm{min}^{-1}$ & $61.6 \pm 3.9$ & $60.1 \pm 4.2$ \\
\hline HR, beats/min ${ }^{-1}$ & $70.8 \pm 2.6$ & $71.4 \pm 2.1$ \\
\hline SBP, mmHg & $114.2 \pm 12.1$ & $112.2 \pm 14.1$ \\
\hline DBP, mmHg & $72.1 \pm 5.1$ & $73.4 \pm 5.8$ \\
\hline
\end{tabular}

a Abbreviations: BMI, body mass index; DBP, diastolic blood pressure; $\mathrm{HR}$, hearth rate; and SBP, systolic blood pressure.

$\mathrm{b}$ Data are presented as mean $\pm \mathrm{SD}$.

c $\mathrm{n}=9$. 
Armanfar Met al.

\begin{tabular}{|c|c|c|c|c|}
\hline Variables & Baseline & 2 & 3 & 4 \\
\hline \multicolumn{5}{|l|}{$\mathbf{L D H}(\mathbf{I U} / \mathbf{L})$} \\
\hline Group Q10 & $320 \pm 8.17$ & $374 \pm 9.87$ & $324 \pm 6.84$ & $329 \pm 3.87$ \\
\hline Placebo group & $320 \pm 7.62$ & $376 \pm 11.12$ & $322 \pm 7.71$ & $378 \pm 4.85$ \\
\hline \multicolumn{5}{|l|}{ MDA, nmol/dL } \\
\hline Group Q10 & $1.49 \pm 0.25$ & $2.85 \pm 0.22$ & $1.54 \pm 0.27$ & $1.72 \pm 0.23^{c}$ \\
\hline Placebo group & $1.51 \pm 0.21$ & $2.88 \pm 0.14$ & $1.58 \pm 0.34$ & $2.95 \pm 0.4$ \\
\hline \multicolumn{5}{|l|}{ TAC, mmol/L } \\
\hline Group Q10 & $0.753 \pm 0.03$ & $0.726 \pm 0.07$ & $0.877 \pm 0.11^{c}$ & $0.862 \pm 0.09^{C}$ \\
\hline Placebo group & $0.742 \pm 0.04$ & $0.699 \pm 0.05$ & $0.710 \pm 0.05$ & $0.708 \pm 0.04$ \\
\hline \multicolumn{5}{|c|}{ Hemoglobin, $\mathrm{g} / \mathrm{dL}$} \\
\hline Group Q10 & $45.5 \pm 0.7$ & $46.6 \pm 0.8$ & $45.7 \pm 0.6$ & $47.1 \pm 0.1$ \\
\hline Placebo group & $46.7 \pm 0.5$ & $46.5 \pm 0.7$ & $45.5 \pm 0.4$ & $46.1 \pm 0.2$ \\
\hline \multicolumn{5}{|l|}{ Hematocrit, \% } \\
\hline Group Q10 & $45.5 \pm 0.7$ & $46.6 \pm 0.8$ & $45.7 \pm 0.6$ & $47.1 \pm 0.1$ \\
\hline Placebo group & $46.7 \pm 0.5$ & $46.5 \pm 0.7$ & $45.5 \pm 0.4$ & $46.1 \pm 0.2$ \\
\hline
\end{tabular}

a Abbreviations: LDH, lactate dehydrogenase; MDA, Malondialdehyde; and TAC, total antioxidation capacity.

b Data are presented as mean \pm SD.

c Significant differences between the two groups.

\section{Discussion}

In the present study, short-term supplementation cause significantly increased TAC baseline levels. Acute supplementation has no significant effect on variables. The reducing exercise-induced changes range of the plasma TAC following the short-term CoQ10 supplementation and attenuated the exercise-induced increase in response of plasma MDA. However, the acute and shortterm CoQ10 supplementation had not significant effect on the exercise-induced increase response of LDH. In normal circumstances blood coenzyme Q levels are not significantly via dietary elements such as dairy products, poultry, eggs and meat. The diets habits of the middle-distance runner participating in the research did not affect the basal levels of antioxidant components in serum. CoQ10 supplementation leads to augment in plasma coenzyme Q concentrations, the extent of which depends upon the type of formulation, dosage, and also duration [17]. The CoQ10 supplement in capsule form was provided in this study at a dosage of $5 \mathrm{mg} / \mathrm{kg} /$ day for 14 days. Previous reports have shown that the bioavailability of CoQ10 can reach maximal concentration at 26.5 or 25.8 hours following supplementation [20]. Thus, daily CoQ10 supplementation could provide maximal concentration in human plasma during experiments. Therefore, CoQ10 supplementation at $5 \mathrm{mg} / \mathrm{kg} /$ day for 14 days was noted to increase plasma CoQ10 levels approximately 2-fold compared to before supplementation. Previous reports suggest that CoQ10 in the ubiquinone form is essential for generating energy within mitochondria and providing antioxidant defense similar to the other fat-soluble anti- oxidants, such as vitamin E. This appears to be due to the scavenging of free radicals and prevention of oxidation of lipids and other molecules [21]. Results from previous animal studies have reported that CoQ10 supplementation increased total CoQ level in various tissues including skeletal muscle in rats $[15,16]$.

CoQ10 plays various critical roles in metabolism, serves as a redox electron transporter in the mitochondria related to the synthesis of ATP, acting as an essential antioxidant, influencing the stability of membranes [3]. Vigorous exercise increases energy requirement manifold and oxidative stress in tissues, and induces muscle damage. Thus, CoQ10 in serum or plasma may have been distributed to several tissues during intensive exercise. Previous studies have suggested that CoQ10 supplementation reduced increased creatine kinase (CK) and lactate dehydrogenase (LDH) in rat's subsequent downhill running [3, 11]. Moreover, some studies have reported that CoQ10 protected cultured skeletal muscle cells from electrical stimulation-induced LDH release. In addition, exogenous administration of CoQ10 suppressed hepatic oxidative damage after reperfusion following ischemia $[3,22]$. Ubiquinone supplementation has the potential to decrease severe activity-induced oxidative stress and muscle injury. Many investigators have showed that exercise increases serum CK activity, which is the most commonly used indicator of skeletal muscle injury induced by exercise [23]. In this study, serum LDH activity in exercise significantly increased about compared with rest. This result indicated that muscle damage was induced 
by intensive exercise. In addition, serum LDH levels were significantly lower in exercise-CoQ10 group compared with placebo group. Therefore, short-term ubiquinone supplementation provided protection against intensive exercise-induced muscular damage. In earlier studies, it has been reported that CoQ10 had a structural stabilizing effect on cell membrane phospholipids [24]. Therefore, it is quite likely that CoQ10 supplementation increases CoQ concentration in muscle cell membranes and reduces strenuous exercise-induced muscular injury by enhancing cell membrane stabilization.

Increases in plasma MDA levels after exercise are widely shown in the literature [17]. The training and competition sessions resulted in increased basal oxidative stress as indicated by the increased MDA plasma levels [17]. The CoQ10 supplementation did prevent increased oxidative stress in Q10 groups. It has been previously reported that an antioxidant supplementation with vitamin C, E and carotene decreased the lipoperoxide levels in basketball players $[17,23]$. Differences in the supplementation, in the oxidative stress markers analyzed and in the competition and training sessions developed by the sportsmen could explain the differences in the results obtained. The molecular damage produced by ROS is parallel to the activation of the endogenous antioxidant defense's [17, 24, 25]. In a similar way, free radicals could be involved in the muscle adaptations to exercise in skeletal muscle; some ROS production is needed to attain optimal muscular isometric force production $[17,26]$. The basal plasma molecular damage increased during the study; this increase could be related with the muscle adaptations to exercise mediated by ROS. The surplus intake of antioxidants with the supplement did not influence the adaptations to exercise $[17,26]$.

DT-diaphorase is an inducible antioxidant enzyme that maintains the reduced antioxidant form of CoQ10 in membrane systems and to protect against xenobiotics which could generate ROS [17, 27]. Recent studies reported that antioxidant supplementation could prevent endogenous antioxidant adaptations to increased ROS production [17, 24]. However, it has been also indicated that molecular damage produced by ROS is parallel to the activation of the endogenous antioxidant defences. Because a similar increase in basal plasma MDA levels had been found in both groups after the 3 months of supplementation in the present study, we could suppose that DT-diaphorase activity could be increased in both groups in parallel to increased MDA levels as a result of regular exercise as it has been indicated previously $[28,29]$.

In addition, we found a significant increase in the TAC in CoQ10 group compared with the placebo group. These results in the TAC are due to the supplementation with an antioxidant substance such as CoQ10, which increases its concentration in plasma as it has been shown in other studies [30].

In summary, Q10 supplementation of the diet for 14 days with dosage $5 \mathrm{mg} / \mathrm{kg} /$ day prevented the stress oxida- tive and muscle damage induced by a running $3000 \mathrm{~m}$ competition without influencing the antioxidant adaptations induced by exercise.

\section{Acknowledgements}

This study carried out in the faculty of physical education and sport sciences in university of Tabriz. This paper is based on a thesis submitted by Mostafa Armanfar to the Ministry Education, in partial fulfillment of the requirement for the MSc degree in physiology from university of Tabriz, Tabriz, Iran.

\section{Authors' Contributions}

All authors declare that they have no conflict of interest.

\section{Conflict of Interest}

The authors declare no conflict of interest.

\section{Funding/Support}

This paper had been done by personal expenses.

\section{References}

1. Liu CC, Huang CC, Lin WT, Hsieh CC, Huang SY, Lin SJ, et al. Lycopene supplementation attenuated xanthine oxidase and myeloperoxidase activities in skeletal muscle tissues of rats after exhaustive exercise. BrJ Nutr. 2005;94(4):595-601.

2. Wu HJ, Chen KT, Shee BW, Chang HC, Huang YJ, Yang RS. Effects of $24 \mathrm{~h}$ ultra-marathon on biochemical and hematological parameters. World J Gastroenterol. 2004;10(18):2711-4.

3. Kon M, Kimura F, Akimoto T, Tanabe K, Murase Y, Ikemune S, et al. Effect of coenzyme Q10 supplementation on exercise-induced muscular injury of rats. Exerc Immunol Rev. 2007;13:76-88.

4. Powers SK, Jackson MJ. Exercise-induced oxidative stress: cellular mechanisms and impact on muscle force production. Physiol Rev. 2008;88(4):1243-76.

5. Diaz-Castro J, Guisado R, Kajarabille N, Garcia C, Guisado IM, de Teresa C, et al. Coenzyme Q(10) supplementation ameliorates inflammatory signaling and oxidative stress associated with strenuous exercise. Eur J Nutr. 2012;51(7):791-9.

6. Vina J, Gomez-Cabrera MC, Lloret A, Marquez R, Minana JB, Pallardo FV, et al. Free radicals in exhaustive physical exercise: mechanism of production, and protection by antioxidants. IUBMB Life. 2000;50(4-5):271-7.

7. Jackson M. Antioxidants, reactive oxygen and nitrogen species, gene induction and mitochondrial function. Mol Aspects Med. 2002;23(1-3):209-85.

8. Kumar CT, Reddy VK, Prasad M, Thyagaraju K, Reddanna P. Dietary supplementation of vitamin $\mathrm{E}$ protects heart tissue from exerciseinduced oxidant stress. Mol Cell Biochem.1992;111(1-2):109-15.

9. Mastaloudis A, Morrow JD, Hopkins DW, Devaraj S, Traber MG Antioxidant supplementation prevents exercise-induced lipid peroxidation, but not inflammation, in ultramarathon runners. Free Radic Biol Med. 2004;36(10):1329-41.

10. Kaikkonen J, Tuomainen TP, Nyyssonen K, Salonen JT. Coenzyme Q10: absorption, antioxidative properties, determinants, and plasma levels. Free Radic Res. 2002;36(4):389-97.

11. Shimomura Y, Suzuki M, Sugiyama S, Hanaki Y, Ozawa T. Protective effect of coenzyme Q10 on exercise-induced muscular injury. Biochem Biophys Res Commun. 1991;176(1):349-55.

12. Zhou S, Zhang Y, Davie A, Marshall-Gradisnik S, Hu H, Wang J, et al Muscle and plasma coenzyme Q10 concentration, aerobic power and exercise economy of healthy men in response to four weeks of supplementation. J Sports Med Phys Fitness. 2005;45(3):337-46.

13. Cooke M, Iosia M, Buford T, Shelmadine B, Hudson G, Kerksick C, et al. Effects of acute and 14-day coenzyme Q10 supplementation 


\section{Armanfar Met al.}

on exercise performance in both trained and untrained individuals. J Int Soc Sports Nutr. 2008;5:8.

14. Dwyer GB, Davis SE. ACSM's health related physical fitness as sessment manual. 4th ed. Philadelphia: Lippincott Williams \& Wilkins; 2008.

15. Gibson RS. Principles of nutritional assessment. 2nd ed. London: Oxford University Press; 2005.

16. Leelarungrayub D, Sawattikanon N, Klaphajone J, Pothongsunan P, Bloomer RJ. Coenzyme Q10 Supplementation Decreases Oxidative Stress and Improves Physical Performance in Young Swimmers: A Pilot Study. Open Sport Med J. 2010;4(1):1-8.

17. Tauler P, Ferrer MD, Sureda A, Pujol P, Drobnic F, Tur JA, et al. Supplementation with an antioxidant cocktail containing coenzyme Q prevents plasma oxidative damage induced by soccer. EurJ Appl Physiol. 2008;104(5):777-85.

18. Bloomer RJ, Goldfarb AH, Wideman L, McKenzie MJ, Consitt LA. Effects of acute aerobic and anaerobic exercise on blood markers of oxidative stress. J Strength Cond Res. 2005;19(2):276-85.

19. American College of Sports Medicine. ACSM's health-related physical fitness assessment manual.Philadelphia: LWW; 2013.

20. Turunen M, Olsson J, Dallner G. Metabolism and function of coenzyme Q. Biochim Biophys Acta. 2004;1660(1-2):171-99.

21. Kagan V, Serbinova E, Packer L. Antioxidant effects of ubiquinones in microsomes and mitochondria are mediated by tocopherol recycling. Biochem Biophys Res Commun. 1990;169(3):851-7.

22. Portakal O, İnal-Erden M. Effects of pentoxifylline and coenzyme Q10 in hepatic ischemia/reperfusion injury. Clinl Biochem. 1999;32(6):461-6.
23. Schroder H, Navarro E, Tramullas A, Mora J, Galiano D. Nutrition antioxidant status and oxidative stress in professional basketball players: effects of a three compound antioxidative supplement. Int J Sports Med. 2000;21(2):146-50.

24. Gomez-Cabrera MC, Borras C, Pallardo FV, Sastre J, Ji LL, Vina J. Decreasing xanthine oxidase-mediated oxidative stress prevents useful cellular adaptations to exercise in rats. $J$ Physiol. 2005;567(Pt 1):113-20.

25. Jackson MJ. Free radicals in skin and muscle: damaging agents or signals for adaptation? Proc Nutr Soc. 1999;58(3):673-6.

26. Reid MB. Invited review: redox modulation of skeletal muscle contraction: what we know and what we don't. J Appl Physiol (1985). 2001;90(2):724-31.

27. Goto S, Radak Z. Hormetic effects of reactive oxygen species by exercise: a view from animal studies for successful aging in human. Dose Response. 2009;8(1):68-72.

28. Radák Z, Kaneko T, Tahara S, Nakamoto H, Ohno H, Sasvári M, et al. The effect of exercise training on oxidative damage of lipids, proteins, and DNA in rat skeletal muscle: evidence for beneficial outcomes. Free Radic Biol Med. 1999;27(1-2):69-74.

29. Radak Z, Sasvari M, Nyakas C, Pucsok J, Nakamoto H, Goto S. Exercise preconditioning against hydrogen peroxide-induced oxidative damage in proteins of rat myocardium. Arch Biochem Biophys. 2000;376(2):248-51.

30. Ochoa JJ, Quiles JL, Huertas JR, Mataix J. Coenzyme Q10 protects from aging-related oxidative stress and improves mitochondrial function in heart of rats fed a polyunsaturated fatty acid (PUFA)rich diet. J Gerontol A Biol Sci Med Sci. 2005;60(8):970-5. 